\title{
Tendencias sociales de la enfermedad de Chagas para las próximas décadas
}

\author{
Social tendencies of Chagas disease in the next \\ decades
}

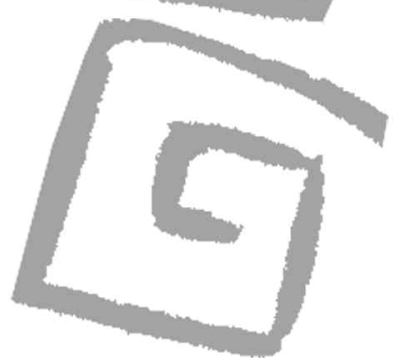

Pinto Dias, João Carlos 1

${ }^{1}$ Médico. Doctor en Medicina Tropical. Miembro Titular, Comité de Enfermedades Olvidadas, Organización Mundial de la Salud. Investigador Emérito, Centro de Pesquisas René Rachou, Fundação Oswaldo Cruz, Brasil. jcpdias@cpqrr.fiocruz.br
RESUMEN La existencia de la enfermedad de Chagas humana se considera el resultado de un conjunto complejo de determinantes bioecológicos y sociales. Su cumbre de incidencia se produjo entre los años 1950 y 1970, observándose una tendencia a la disminución en las décadas siguientes. Innumerables elementos socioculturales y políticos están presentes también en la expansión geográfica de la enfermedad, en su prevención y en la atención médica a los infectados. Para las próximas décadas se espera una mayor reducción de la transmisión, especialmente vectorial y transfusional, como producto de las acciones de control y de los cambios en el sistema de producción, en paralelo con la urbanización y con las acciones antrópicas extensivas en las áreas endémicas. Se espera también una reducción en la morbilidad, dependiente de un mejor acceso a los sistemas de salud y de los constantes avances en la medicina. Sin embargo, la enfermedad seguirá siendo muy importante por dos o tres décadas más, dejando como principales desafíos la vigilancia sobre su transmisión y la atención a los individuos ya infectados. Está prevista una disminución en la visibilidad de la enfermedad, con reflejos negativos en la prioridad política de las acciones sobre su atención y control.

PALABRAS CLAVE Enfermedad de Chagas; Condiciones Sociales; Epidemiología/ tendencias.

ABSTRACT Chagas disease is known to be the result of a complex set of bio-ecological and social determinants. The maximum incidence of the disease occurred between 1950 and 1970, with a downward tendency observed in the following decades. Innumerable socio-cultural and political elements take part in the geographic expansion of the disease, the prevention of the disease and in the medical attention given those infected. Over the next decades a major reduction in transmission is expected, especially in the vector and transfusion routes, as a consequence of control-oriented programs and changes in the production system, in conjunction with urbanization processes and extensive anthropic actions in endemic areas. A reduction in morbidity is also expected, owing to better access to the health system and to medical advances. Nevertheless, Chagas disease will continue to have great importance in the next two to three decades, the main challenges being surveillance of disease transmission and the medical attention provided to infected individuals. A reduction in the visibility of the disease, with negative repercussions in the political priority given to medical attention and disease control, is also anticipated.

KEY WORDS Chagas Disease; Social Conditions; Epidemiology/trends. 


\section{INTRODUCCIÓN}

La enfermedad de Chagas humana $(\mathrm{ECH})$ existe en el Nuevo Mundo hace por lo menos nueve mil años. Es una entidad endémica latinoamericana derivada de una enzootia silvestre mucho más antigua, en la que el protozoario Trypanosoma (Schizotrypanum) cruzi (T. cruzi) circula en diferentes ecotopos, entre vectores invertebrados (Hemiptera, Reduviidae, Triatominae) y mamíferos de pequeño porte (1-5). Esta enfermedad posee determinantes sociales, económicos y políticos muy claros y fundamentales que dependen, por un lado, de la ocupación por parte del hombre de ambientes naturales en la búsqueda de supervivencia y riqueza y, por otro, de la pobreza, de las acciones antrópicas y las malas relaciones de producción (6). Su dispersión se produjo a través de los movimientos poblacionales internos en América Latina, desde las migraciones ancestrales de pueblos nativos como los quechuas, los aztecas y los mayas, intensificándose al extremo con la invasión europea posterior a Colón $(3,7)$. Los hallazgos socioculturales y políticos de tal expansión, muy vinculada con la vivienda rural precaria, han sido retratados por distintos autores $(3,6,8,9)$. La cumbre epidemiológica de la $\mathrm{ECH}$ se da al inicio de la segunda mitad del siglo $X X$, afectando sobre todo a las poblaciones socialmente deprimidas de los ambientes campesinos continentales, desde México a la Patagonia. Desde entonces, los cambios en el sistema de producción, las olas de urbanización y las acciones de control (vectorial y transfusional) fueron reduciendo la incidencia, la prevalencia y el impacto médico-social de la enfermedad $(2,10,11,12)$.

En el presente texto se analizan algunas tendencias perceptibles o estimadas de la $\mathrm{ECH}$ para los próximos veinte años, enfocando particularmente los aspectos sociales ligados a su producción, expansión, manejo médico y control.

\section{PERFIL ACTUAL DE LA ENFERMEDAD DE CHAGAS HUMANA Y CAMBIOS OCURRI- DOS EN LAS TRES ÚLTIMAS DÉCADAS}

La reducción de la incidencia de la ECH, observada en la década de 2010 , se debió principalmente al control vectorial y de los bancos de sangre, intensificado desde los años 1970 en varias regiones endémicas. Con la disminución de los casos nuevos y la pérdida de infectados por muerte (especialmente debida a la cardiopatía crónica avanzada), se observó también una reducción progresiva de la prevalencia en varias regiones $(13,14)$, quedando algunos residuos de transmisión en áreas más pobres y no controladas (o controladas de un modo insuficiente) como, por ejemplo, en extensas áreas del Chaco Sudamericano y partes de México. Las dos principales especies transmisoras del vector (Triatoma infestans y Rhodniusprolixus) han sido virtualmente eliminadas de muchas regiones de dispersión original, mientras que la proporción de bancos de sangre bajo control serológico asciende a más del $95 \%$ en la mayoría de los países endémicos. Por otro lado, está ocurriendo una reducción de las transmisiones congénita y transfusional debido a que ha disminuido la frecuencia de infecciones, tanto en mujeres embarazadas jóvenes como en donantes de sangre. En las dos últimas décadas, se observa además un descenso en la morbilidad y en la mortalidad de la $\mathrm{ECH}$ en muchas áreas continentales, especialmente en aquellas bajo control de transmisión y con mejores coberturas médico-asistenciales $(14,15)$. En contraposición, se han detectado episodios de transmisión oral en áreas con presencia de triatominos silvestres, especialmente en la región amazónica, en números que oscilan entre 100 y 200 casos/año, aunque aún subdimensionados $(2,14,16,17)$. También es preocupante la intensificación de las migraciones de infectados crónicos desde América Latina hacia países no endémicos de prácticamente todo el mundo $(2,12,15)$. En resumen, los principales cambios epidemiológicos y político-sociales ocurridos con relación a la $\mathrm{ECH}$ en las últimas dos o tres décadas han sido: la evolución global de las relaciones y tecnologías de producción agropecuaria, la fuerte tendencia urbano-industrial, la acentuación de las migraciones rural-urbana e internacionales, la expansión de los programas de control y los avances técnicos en el manejo médico de los infectados. Al respecto, el Cuadro 1 muestra algunas cifras significativas presentadas por el Dr. Roberto Salvatella, con estimaciones de la Organización Panamericana de la Salud. 
Cuadro 1. Cambios en los parámetros epidemiológicos de la enfermedad de Chagas en las Américas. Años 1990, 2000 y 2006.

\begin{tabular}{llll}
\hline Parámetros epidemiológicos & $\mathbf{1 9 9 0}$ & $\mathbf{2 0 0 0}$ & $\mathbf{2 0 0 6}$ \\
\hline Muertes anuales & $>45.000$ & 21.000 & 12.500 \\
Casos humanos de infección & 30 millones & 18 millones & 15 millones \\
Nuevos casos anuales & 700.000 & 200.000 & 41.200 \\
Población en riesgo & 100 millones & 40 millones & 28 millones \\
Distribución & 21 países & 19 países & 18 países \\
& & & \\
\hline
\end{tabular}

Fuente: Salvatella (18), sobre estimaciones del Special Programme for Research and Training in Tropical Diseases (TDR), World Health Organization.

En la actualidad, la extensión de la ECH se ha reducido fundamentalmente a las áreas endémicas, donde la mortalidad todavía es elevada. Por otra parte, se estima que la prevalencia no supera los 7-8 millones de infectados y que la incidencia es aún más reducida $(14,19)$. En términos socioeconómicos, los cálculos del Banco Mundial han mostrado una significativa reducción de la pérdida de días productivos/año por la $\mathrm{ECH}$ entre 1990 y 2007 (20).

La herencia y los desafíos actuales de la ECH están basados en tres pilares:

a) Implementar, expandir y consolidar programas de control vectorial en los países endémicos, que incluya vigilancia epidemiológica sostenible (21).

b) Implementar, expandir y consolidar programas para el control de bancos de sangre, ampliado adecuadamente para países no endémicos que reciben inmigración de infectados $(12,22)$.

c) Proveer atención médica y seguridad social para todos los infectados, que incluya prospección (y tratamiento, si fuera necesario) de niños hijos de madres seropositivas, incluso de las poblaciones más alejadas y aisladas $(2,23,24)$.

\section{ASPECTOS SOCIALES DE LA ENFERMEDAD DE CHAGAS HUMANA: TENDENCIAS ESPERABLES EN LOS PRÓXIMOS AÑOS}

El escenario de proyección más plausible se ubica entre los próximos veinte y treinta años, al considerar pragmáticamente la historia natural de la enfermedad, los lentos cambios ecológicos, sociales y políticos en las áreas endémicas, la evolución científica y el proceso sociopolítico de la globalización $(12,15)$. Los parámetros para la reflexión serán los epidemiológicos tradicionales (transmisión, incidencia, prevalencia, morbilidad y peso médico social).

La transmisión vectorial de la $\mathrm{ECH}$ tiene mucho que ver con los hechos socioculturales y políticos de una región endémica $(6,21)$. Su tendencia seguirá siendo descendente, en virtud de los programas de control, de las acciones antrópicas extensivas sobre el ambiente, de la migración rural urbana y de fuertes cambios en el sistema de producción agropecuario. Sin embargo, la vía "vectorial/oral" se mantendrá en sus niveles actuales en algunas áreas (por ejemplo, el estado de Pará en Brasil), con posibilidad de casos eventuales en cualquier otra área en donde se encuentren triatominos silvestres $(2,7)$. Las principales acciones humanas sobre el ambiente relativas a la $\mathrm{ECH}$ son fundamentalmente los monocultivos, la deforestación y la construcción de carreteras, ciudades y centrales hidroeléctricas, las que conllevan a la disminución de los vectores nativos en el ambiente natural $(6,25)$. Sin embargo, la deforestación puede funcionar, en primera instancia, como impulso para la invasión de ecotopos artificiales por especies nativas, principalmente en busca de abrigo y alimento, o también por atracción luminosa, proporcionando eventuales episodios de transmisión oral dependiente del vector. Ya el fenómeno de la colonización intradomiciliaria de esas especies es muy raro, lento y dependiente de características bio- 
ecológicas, al carecer en general, de largos períodos de tiempo para su instalación o recuperación $(25,26,27)$. En este sentido, son muy típicos los eventos ocurridos en los últimos cuarenta años en la Amazonia brasileña: la marcada tendencia a las migraciones internas y la deforestación masiva dieron margen a una preocupación de los científicos y salubristas con respecto a la incidencia de casos nuevos de la $\mathrm{ECH}$ en la región, motivada por la domiciliación de vectores nativos y el transporte pasivo de especies alóctonas, en particular $T$. infestans y $T$. brasiliensis $(16,26,28)$. Afortunadamente, esta situación no se trasladó hasta el presente, dadas las características ecológicas del área y la baja valencia ecológica de los vectores nativos $(15,27,29,30)$. A su vez, ciertos elementos de evolución social, como el fenómeno de la urbanización, los programas de control de vectores y las progresivas mejoras de las viviendas rurales (por ejemplo, la introducción de cocinas a gas) produjeron una significativa reducción de especies introducidas y secundarias en el ambiente humano más cercano $(10,31)$ Al observar la gran influencia de la globalización y de los cambios en la producción sobre la transmisión vectorial, en un análisis reciente se destacan los siguientes aspectos (15):

- La deforestación resultante de un fuerte mercado de maderas, de la expansión de las agroindustrias y de la apertura de nuevas fronteras agrícolas en las áreas endémicas.

- El empleo extensivo de pesticidas en los modernos proyectos agroindustriales.

- La expansión del uso de electricidad y de maquinaria industrial que influye sobre el comportamiento de los vectores y la demografía rural.

- La progresiva reducción de reservorios naturales del parásito, como producto de la deforestación, de los pesticidas y del monocultivo extensivo.

- Los cambios en el modelo de producción y el mercado dominado por economías de escala, con prioridad para las agroindustrias por sobre la estrategia clásica de la economía.

- La implementación de factores de modernización, especialmente de automatización, robotización y de mejoramiento de viviendas.
La acción antrópica sobre el ambiente tiende a crecer en todo el mundo, con ciertas particularidades en el área originalmente endémica de la $\mathrm{ECH}(25,26)$. La búsqueda de producción primaria agropecuaria, así como la búsqueda de energía limpia, son necesidades mundiales urgentes, que ya afectan y tienden a crecer en Latinoamérica. El cultivo extensivo de alimentos con tecnología moderna progresa en áreas pobres y clásicamente endémicas para la $\mathrm{ECH}$, como en el Cerrado brasileño (café, caña, soya, ganadería extensiva), en el Chaco Sudamericano (soya, caña) y el Noreste árido de Brasil (caña, frutas). Estas actividades exigen fuertes capitales externos, expulsan a las poblaciones pobres y reducen los nichos naturales para vectores $y$ reservorios. No hay una tendencia hacia la supervivencia o un retorno al modelo antiguo de las microeconomías familiares. Las demandas del mercado y los determinantes políticos y económicos de la globalización son las razones fundamentales de este proceso (15). Los casos residuales de transmisión vectorial, como ya fue mencionado, deben disminuir progresivamente, focalizándose en sitios más pobres y aislados del continente, en donde la vigilancia y el control de la ECH seguirán siendo dependientes de políticas públicas coherentes y sostenidas $(10,21)$. Sobre el tema asociado de la vivienda humana como reducto de vectores y transmisión de la $\mathrm{ECH}$, la tendencia es de progresiva disminución del rancho de mala calidad, sobre todo como resultado de los procesos de urbanización y de modernización de la producción agropecuaria. Este proceso será más lento en microrregiones de pobreza como en el Chaco Sudamericano y en el noreste de Brasil. En el marco macropolítico, no se esperan ampliaciones en los programas gubernamentales de vivienda rural en la gran mayoría de las áreas endémicas, dado que la tendencia actual es la urbanización y las prioridades políticas en este sentido están netamente vinculadas a los programas de viviendas populares urbanas $(32,33)$. Como detalle epidemiológico, los residuos principales de vectores tienden a concentrarse en el peridomicilio, más accesible a las especies secundarias nativas y con más dificultades para el control químico $(10,30)$. La cría de gallinas es habitual en el medio rural y los gallineros constituyen, en general, el principal foco peridomiciliario de 
triatominos. Por lo tanto, la producción masiva de pollos en el ámbito de la globalización podría ocasionar una importante tendencia a la disminución de gallineros en las casas. Lo mismo se aplica a otros animales de cría doméstica como fuente familiar de alimentos, como el conejo, el cuy, etc. $(27,32)$.

Al considerar en paralelo la transmisión oral, especialmente como un producto particular de la presencia de vectores infectados en la proximidad del hombre y de sus alimentos, es aún imprevisible pronosticar su tendencia, aunque en la región amazónica se encuentra más concentrada. Sin embargo, se puede admitir una tendencia lenta de reducción, en la medida que progresan los fenómenos de industrialización y mejoran las prácticas de preparación de comidas $(2,17,29)$.

Para la transmisión transfusional, la tendencia es de una virtual eliminación, en el cortomediano plazo. En su base está el control serológico activo de donantes de sangre, cada vez más universalizada y de mejor calidad, reforzado por la entrada de cada vez menos infectados (especialmente jóvenes) al sistema de donación $(7,10)$. En Brasil, por ejemplo, de una estimación de 15.000 casos de transmisión transfusional en los años 1970, hoy en día no se esperan más de 10 casos anuales $(3,22)$. En el plano socio-políticoadministrativo habrá en el futuro un momento de definición respecto a la continuidad de la serología para Chagas en bancos de sangre, dado que se gasta mucho en miles y millones de análisis para encontrar un mínimo de donantes infectados $(3,10,11)$. Sin embargo, el control del HIV transfusional seguirá siendo el principal argumento para el mantenimiento de una rigurosa vigilancia (sanitaria y epidemiológica) en los bancos de sangre de todo el mundo, una situación que aún debe perdurar por algunas décadas y que trae beneficios para el control del Chagas, de la sífilis y de las hepatitis virales $(11,22)$. En el ámbito médico-social, hay que perfeccionar la derivación de los sujetos seropositivos hacia un sistema capacitado de atención de la $\mathrm{ECH}$, lo que no ocurre regularmente en todas partes, incluso en países no endémicos $(7,10,11,22)$.

Para la transmisión congénita, aunque sea muy compleja su prevención en términos médicos, la tendencia es obviamente hacia su progresiva eliminación, en la medida en que se controlen las otras formas de transmisión. Una demostración de esto puede encontrarse en muchas encuestas de seroprevalencia entre mujeres embarazadas en regiones bajo control vectorial y transfusional, con residuos de seropositividad solo en edades por encima de los 30 años (10,34-36). El Cuadro 2 muestra la incidencia de casos congénitos en Brasil en el año 2010.

Estas estimaciones (Cuadro 2) no están lejos de la realidad, dado que las recientes encuestas serológicas entre niños pequeños en el país, solo han detectado muy excepcionalmente a individuos seropositivos. Por ejemplo, en la última encuesta nacional, en edades menores de cinco años, entre casi 105 mil sueros obtenidos en todo el país solo 32 han sido positivos $(0,03 \%)$, con un máximo de 20 casos atribuibles a transmisión congénita (36). Desde el punto de vista asistencial, aún existen pocos programas regulares y continuos de diagnóstico y seguimiento del binomio madre infectada/niño, con excepciones en Uruguay y partes de Argentina, Paraguay y Brasil, con posibilidades reales de curar a un recién nacido infectado. Depender solo de la detección clínica de los casos congénitos para establecer la atención es muy poco efectivo en términos de Salud Pública, dado que la mayoría de los casos son asintomáticos $(7,35)$. Al igual que en el control vectorial, la tendencia de las autoridades sanitarias es invertir cada vez menos en programas de atención al Chagas congénito en países como Brasil, donde su ocurrencia es mínima (15).

Cuadro 2. Estimación de la incidencia de casos congénitos de la enfermedad de Chagas humana en Brasil, año 2010.

\begin{tabular}{|c|c|c|}
\hline Evento & Número & $\%$ \\
\hline Nacimientos vivos & 2.920 .603 & 100,00 \\
\hline Madres 15-29 añosa & 2.587 .477 & 88,60 \\
\hline Madres 30-59añosb & 332.831 & 11,40 \\
\hline Total de madres seropositivas & 7.837 & 0,27 \\
\hline Riesgo estimado de transmisiónc & - & 0,60 \\
\hline
\end{tabular}

Fuente: Elaboración propia a partir de datos del Departamento de Informática do Sistema Único de Saúde (DATASUS).

a Infección estimada 0,2\%: 5.174 madres seropositivas.

b Infección estimada 0,8\%: 2.663 madres seropositivas.

c 47 casos en el año. 


\section{Incidencia y prevalencia}

La incidencia y la prevalencia son parámetros dependientes de los niveles de transmisión ya discutidos. La tendencia general es obviamente regresiva, manteniéndose estable en aqueIlos sitios sin acciones de control $(10,24)$. Para la prevalencia se añade el factor mortalidad, cada vez más significativo, a medida que los individuos infectados van envejeciendo, agregándose muertes por muchas otras causas crónico-degenerativas $(10,33,37-40)$.

\section{Morbilidad}

La morbilidad de la ECH corresponde sobre todo a la fase crónica, especialmente en términos de cardiopatías $(9,41,42)$. Hasta un $10 \%$ de los agudos suelen morir en el caso de que no sean tratados oportunamente, lo que amerita un rápido diagnóstico. El tratamiento del agudo presenta una mayor proporción de curas parasitológicas, lo que resulta en un mejor pronóstico para el paciente a largo plazo (34). Además, el individuo crónico suele beneficiarse con una buena atención médica (incluyendo el tratamiento específico en los casos indicados), con mejora en la calidad y la cantidad de vida $(9,11,34,42,43)$. Todo esto presupone una capacidad médico-asistencial instalada, especialmente en zonas endémicas con transmisión activa, lo que desafortunadamente se encuentra en situación muy débil. Es reconocido que en Brasil y en otros países hay cada vez menos expertise médica sobre la ECH y que los currículos de las facultades de medicina, en general, tienden a reducirse en relación con este tema $(15,44)$. Por otro lado, con la evolución de la medicina en general, muchos fármacos y procedimientos diagnósticos y terapéuticos destinados a otros agravios van siendo aprovechados en la $\mathrm{ECH}$, muy especialmente en la cardiopatía crónica, lo que posibilita considerables avances en su manejo clínico. Como ejemplo pueden mencionarse los modernos antiarrítmicos, los cardiodesfibriladores, los inhibidores de la enzima convertidora de la angiotensina (ECA), las técnicas quirúrgicas modernas del aparato digestivo, el aumento de indicaciones para el tratamiento específico, etc. $(2,23,43,45)$.

\section{Atención médica y social}

El infectado crónico con cardiopatía o megas constituye el problema fundamental de la atención médica de la $\mathrm{ECH}$, por sus pérdidas de calidad y cantidad de vida. En su gran mayoría, es un individuo pobre que financiera, intelectual y políticamente depende del Estado para su supervivencia y su atención médica $(6,33)$. Los elementos fundamentales involucrados en el problema de la atención al "chagásico" pueden resumirse en acceso, cobertura, expertise, adherencia, referencia y contrarreferencia (45). Existe un esfuerzo general de mejorar la atención médica al infectado en países endémicos y no endémicos, con apoyo de agencias diversas como la Organización Mundial de la Salud (OMS), la Organización Panamericana de la Salud (OPS), Médicos Sin Fronteras, etc. Sin embargo, la formación de profesionales capacitados en las universidades es precaria, con tendencia a la disminución, en vista del progresivo desinterés académico por la ECH (15). En particular, los aspectos políticoadministrativos relativos a los sistemas nacionales de salud vienen adoptando distintas perspectivas en la atención al infectado (46). De manera general, la tendencia a la descentralización de los sistemas de salud, la urbanización creciente y un relativo incremento del acceso del infectado a los servicios médicos pueden ser considerados como hechos positivos para el nuevo milenio (15). En Brasil, por ejemplo, las nuevas políticas sociales puestas en práctica durante los últimos 17 años buscan ofrecer un sistema de salud universal con equidad -Sistema Único de Salud (SUS)-, ofreciendo además fármacos e intervenciones para aquellos que no pueden pagarlos, como por ejemplo la implantación gratuita de marcapasos, así como la disponibilidad sin costos de benznidazol, amiodarona, diuréticos e inhibidores de la ECA, etc. Sin embargo, incluso con los avances observados, el SUS necesita aumentar su resolución en términos de un acceso más rápido, mejores contrarreferencias y expertise, lo que amerita la decisión por parte del gobierno y un estímulo continuo por parte de la comunidad científica (33). 


\section{Aspectos macro políticos y el rol de la comunidad científica}

Con todas sus principales características sociales y geográficas, la ECH puede considerarse como un problema del Estado, tanto en áreas endémicas como en las no endémicas. Su control depende de leyes, de voluntad política y de acciones específicas de salud pública, técnicamente adecuadas. Las acciones de los gobiernos basadas en la descentralización de los servicios de salud y el progresivo achicamiento de los Estados -marca de las dos últimas décadas- pueden ser elementos positivos o negativos en la lucha contra el Chagas dependiendo de su continuidad y grado de cobertura. Aunque la tendencia es de disminución y pulverización de las acciones de control, por falta de articulación, de expertise y de prioridad política de una enfermedad de pobres. Algo similar sucede en los países no endémicos, en donde la atención a la salud de los infectados migrantes sufre la influencia negativa de su condición de pobreza y clandestinidad $(6,8)$. En este contexto, es clásica la posición de la comunidad científica dedicada al Chagas, con sus constantes apelaciones políticas a sus gobiernos, impulsando incluso las articulaciones supranacionales de control (iniciativas intergubernamentales) $(33,46)$.

\section{REFLEXIONES FINALES}

Entre las tendencias demográficas económicas y de epidemiología social de las próximas décadas seguirán ocurriendo cambios importantes, que impactarán sobre la enfermedad. La dispersión de las personas infectadas hacia los centros urbanos y países no endémicos se mantendrá de manera estable aún por algunos años, con una posible tendencia regresiva a largo plazo. Los factores clave son la búsqueda de empleos y la falencia de los modelos antiguos de producción rural. En la medida que se produzca un vaciamiento de la población rural y un avance en los programas de control se reducirá progresivamente el flujo de infectados (15). La disminución de vectores domiciliados y el control de los bancos de sangre, al reducir la transmisión y la visibilidad de la $\mathrm{ECH}$, provocarán una pérdida de prioridad en las acciones de control, lo que será más grave en circunstancias de emergencia de otros problemas endémicos como el dengue, las hantavirosis, la influenza, etc., $(6,15,20)$. Los soportes de la educación para las acciones de control y vigilancia desafortunadamente serán cada vez menos fuertes y estarán cada vez menos presentes en las áreas endémicas, por lo que será cada vez más difícil insertar la ECH en los programas de enseñanza formal en los países endémicos $(10,12,15)$. A su vez, con la fuerte tendencia hacia la descentralización en los sistemas nacionales de salud, las acciones de control y vigilancia deberán depender cada vez más de las instancias locales, lo que resultará en un vaciamiento de los niveles centrales de los países $(33,46)$. Todo esto ya está ocurriendo en Brasil, lamentablemente con pérdida de datos y disminución flagrante de las referencias nacionales $(10,13,15)$. Por otro lado, hubo grandes beneficios sociales y políticos derivados de las ya mencionadas iniciativas intergubernamentales de control, que involucraron a países de toda el área endémica, y posibilitaron mejoras técnicas y científicas en paralelo con una efectiva aproximación política entre los implicados (12,32). Sobre el infectado crónico, todo indica que seguirá muy pobre, aislado o migrante, muchas veces clandestino, y dependiente de los servicios públicos de salud. Para la comunidad científica comprometida con la $\mathrm{ECH}$, todo indica que las motivaciones y el interés en seguir investigando deben también reducirse, así como deberá naturalmente bajar el peso social de la endemia en las agencias internacionales de fomento, como la OPS y la OMS. A pesar de todos los avances, la ECH seguirá siendo un problema social muy importante por veinte años o más, así como una fuente de crecimiento científico y político entre los países afectados, con interés también en algunos potencialmente afectados, como EE.UU. (15,33,44,46-48). 


\section{REFERENCIAS BIBLIOGRÁFICAS}

1. Carlier Y, Dias JCP, Luquetti AO, Honteberye $M$, Torrico $F$, Truyens $C$. Trypanosomiase américaine ou maladie de Chagas. Encyclopedie Médico-Chirurgicale. Paris: Elsevier; 2002. SAS 8-505-A-20

2. Coura JR, Dias JCP. Epidemiology, control and surveillance of Chagas disease: 100 years after its Discovery. Memórias do Instituto Oswaldo Cruz. 2009;104(Suppl 1):S31-S40.

3. Dias JCP, Coura JR. Epidemiologia. En: Dias JCP, Coura JR. Clínica e terapêutica da doença de Chagas: Um manual prático para o clínico geral. Rio de Janeiro: Fiocruz; 1997. p. 33-66.

4. Miles MA, Yeo M, Gaunt MW. Epidemiology of American Trypanosomiasis. En: Maudlin I, Holmes PH, Miles MA. The Trypanosomes. London: CABI Publishing; 2004.

5. World Health Organization. Control of Chagas' disease: Report of a WHO Expert Committee. Geneva: WHO; 2002. (Technical Report Series No. 905).

6. Dias JCP, Briceño-León R, Storino R. Aspectos sociales, económicos, políticos culturales y psicológicos. En: Storino R, Milei J. Enfermedad de Chagas. Buenos Aires: Doyma Argentina; 1994. p. 527-548.

7. Dias JCP, Macedo VO. Doença de Chagas. En: Coura JR. Dinâmica das doenças infecciosas e parasitárias. Rio de Janeiro: Guanabara Koogan; 2005. p. 557-593.

8. Briceño-León R. La enfermedad de Chagas y las transformaciones sociales en América Latina. En: La enfermedad de Chagas a la puerta de los 100 años del conocimiento de una endemia americana ancestral. Washington: OPS; 2007. p. 219230. (OPS/CD/426-06).

9. Prata AR. Clinical and epidemiological aspects of Chagas disease. Lancet Infectious Diseases. 2001;1(2):92-100.

10. Dias JCP, Silveira AC, Schofield CJ. The impact of Chagas Disease control in Latin America: A review. Memórias do Instituto Oswaldo Cruz. 2002;97(5):603-612.

11. Schmunis GA. Tripanossomíase americana: seu impacto nas Américas e perspectivas de eliminação. En: Dias JCP, Coura JR, organizadores. Clínica e terapêutica da doença de Chagas: Uma abordagem prática para o clínico geral. Rio de Janeiro: Fiocruz; 1997. p. 11-24.

12. Schmunis GA. Enfermedad de Chagas en un mundo global. En: La enfermedad de Chagas a la puerta de los 100 años del conocimiento de una endemia americana ancestral. Washington: OPS; 2007. p. 251-267. (OPS/CD/426-06).

13. Brasil, Ministério da Saúde, Secretaria de Vigilância Sanitária. Aspectos epidemiológicos da doença de Chagas [Internet]. 2011 [citado 12 jul 2012]. Disponible en: http://portal.saude.gov.br/ portal/saude/profissional/visualizar_texto.cfm?idtxt $=31454$.

14. Organización Panamericana de la Salud. Estimación cuantitativa de la enfermedad de Chagas en las Américas. Washington: OPS; 2006. (OPS/HDM/CD/425-06).

15. Dias JCP, Coura JR. Globalization and Chagas Disease. En: Delic Z, editor. Globalization and responsibility. Rijeka: Intech; 2012. p. 153-166.

16. Coura JR, Junqueira A. Risks of endemicity, morbidity and perspectives regarding the control of Chagas disease in the Amazon Region. Memórias do Instituto Oswaldo Cruz. 2012;107(2):145-154.

17. Pinto AYN, Valente AS, Valente VC, Ferreira Jr AG, Coura JR. Fase aguda da doença de Chagas na Amazônia brasileira: Estudo de 233 casos do Pará, Amapá e Maranhão, observados entre 1988 e 2005. Revista da Sociedade Brasileira de Medicina Tropical. 2008;42(Suppl 2):S103-S105.

18. Salvatella R. Eliminación de las enfermedades desatendidas y otras enfermedades transmisibles relacionadas con la pobreza [Internet]. 2009 [citado 12 jul 2012]. Disponible en: http://new.paho.org/ hq/dmdocuments/2009/07_Elim_Chagas_junio_09 .pdf.

19. Martins-Melo FR, Alencar $\mathrm{CH}$, Ramos AJR, Heukelbach J. Epidemiology of mortality related to Chagas' Disease in Brazil, 1999-2007. PLoS Neglected Tropical Diseases. 2012;6(2):1508. DOI:10.1371/journal.pntd.0001508.

20. Schmunis GA. Chagas'disease in Latin America and its dissemination to developed world. Revista da Sociedade Brasileira de Medicina Tropical. 2009;42(Suppl 2):S29-S35.

21. Schofield CJ, Jannin J. Salvatella R. The future of Chagas disease control. Trends in Parasitology. 2006;22(12):583-588. 
22. Moraes-Souza H, Silva MMF. Control of transfusion transmission of Chagas disease in different Brazilian regions. Revista da Sociedade Brasileira de Medicina Tropical. 2009;42(Suppl 2):S103-S105.

23. Oliveira Jr W. All-around care for patients with Chagas disease: a challenge for the XXI century. Memórias do Instituto Oswaldo Cruz. 2009;104(Suppl 1):S181-S186.

24. Rassi Jr A, Dias JCP, Marin Neto JA, Rassi A. Challenges and opportunities for primary, secondary and tertiary prevention of Chagas' disease. Hearth. 2009;95(7):524-534.

25. Dias JCP. Rural resource development and its potential to introduce domestic vectors into new epidemiological situation. Revista Argentina de Microbiología. 1988;20:81-85.

26. Forattini OP. Biogeografia, origem e distribuição da domiciliação de triatomíneos no Brasil. Revista de Saúde Pública. 1980;15:265299.

27. Schofield CJ. Triatominae: biology \& control. London: Eurocommunica Publication; 1994.

28. Dias JCP, Prata A, Schofield CJ. Doença de Chagas na Amazônia: esboço da situação atual e perspectivas de prevenção. Revista da Sociedade Brasileira de Medicina Tropical. 2002;35(6):669678.

29. Aguillar HM, Abad-Franch F, Dias JCP, Junqueira ACV, Coura JR. Chagas disease in the Amazon Region. Memórias do Instituto Oswaldo Cruz. 2007;102(Suppl 1):S47-S55.

30. Moncayo A, Silveira AC. Current epidemiological trends for Chagas disease in Latin America and future challenges in epidemiology, surveillance and health policy. Memórias do Instituto Oswaldo Cruz. 2009;104(Suppl 1):S31-S40.

31. Zeledón R, Rabinovich JE. Chagas disease: an ecological appraisal with special emphasis on its insect vectors. Annals Review Entomology. 1981;26:101-103.

32. Dias JCP, Schofield CJ. The evolution of Chagas disease (American trypanosomiasis) control after 90 years since Carlos Chagas discovery. Memórias do Instituto Oswaldo Cruz. 1999;94(Suppl 1):S103-S122.

33. Schmunis GA, Dias JCP. La reforma del sector salud, descentralización prevención y control de enfermedades transmitidas por vectores. Cadernos de Saúde Pública. 2000;16(Suppl 2):S117-S123.

34. Dias JCP. Longitudinal studies on human Chagas Disease in Bambuí, Minas Gerais, Brazil. Revista da Sociedade Brasileira de Medicina Tropical. 2009;42(Suppl 2):S61-S72.

35. Carlier Y, Torrico F. Congenital infection with Trypanosoma cruzi: from mechanisms of transmission to strategies for diagnosis and control. Revista da Sociedade Brasileira de Medicina Tropical. 2003;36(6):767-771.

36. Luquetti AO, Passos ADC, Silveira AC, Ferreira AW, Macedo $V$, Prata AR. O inquérito nacional de seroprevalência de avaliação do controle da doença de Chagas no Brasil (2001-2008). Revista da Sociedade Brasileira de Medicina Tropical. 2011;44(Suppl 2):S108-S121.

37. Drumond JAG, Marcopito LF. Migração interna e a distribuição da mortalidade por doença de Chagas, Brasil, 1981-1998. Cadernos de Saúde Pública. 2006;22(10):2131-2140.

38. Freitas HF, Chizzola PR, Paes AT, Lima AC, Mansur AJ. Risk stratification in a Brazilian hospitalbased cohort of 1220 outpatients with heart failure: role of Chagas' heart disease. International Journal of Cardiology. 2005;102(2):239-247.

39. Lima-Costa MF, Matos DL, Ribeiro ALP. Chagas Disease predicts 10-year stroke mortality in community-dwelling elderly the Bambuí cohort study of aging. Stroke. 2010;41:2477-2482.

40. Wanderley DMV, Litvoc J. Doença de Chagas como causa básica de óbito na região sudeste do Brasil: presença de causas contributárias. Revista de Saúde Pública. 1994;28(1):69-75.

41. Laranja FS, Dias E, Nóbrega GC, Miranda A. Chagas' disease (American trypanosomiasis): A clinical, epidemiologic and pathological study. Circulation. 1956;58:1034-1058.

42. Rassi Jr A, Rassi A, Rassi SG. Predictors of mortality in chronic Chagas disease: a systematic review of observational studies. Circulation. 2007;115(8):1101-1108.

43. Brasil, Ministério da Saúde, SVS. Consenso Brasileiro em doença de Chagas. Revista da Sociedade Brasileira de Medicina Tropical. 2005;38(Suppl 3):S1-S29.

44. Dias JCP. Globalização, inequidade e doença de Chagas. Cadernos de Saúde Pública. 2007;23(Suppl 1):S13-S19. 
45. Dias JCP. Prevenção secundária em doença de Chagas. Revista da Sociedade Brasileira de Medicina Tropical. 2001;34(Suppl 3):S24-S36.

46. Dias JCP. Aspectos políticos em doença de Chagas. Revista da Sociedade Brasileira de Medicina Tropical. 1995;28(Suppl 3):S41-S45.
47. Coura JR, Viñas PA. Chagas disease: a new worldwide challenge. Nature. 2010; 465(7301): 56-58.

48. Zeledón R, Beard CB, Dias JCP, Leiby DA, Dorn PL, Coura JR. An appraisal of the status of Chagas Disease in the United States. London: Elsevier; 2012.

\section{FORMA DE CITAR}

Pinto Dias JC. Tendencias sociales de la enfermedad de Chagas para las próximas décadas. Salud Colectiva. 2012;8(Supl 1):S39-S48.

Recibido el 23 de agosto de 2012

Aprobado el 18 de septiembre de 2012 